\title{
Device for the study of sharp peaks behavior under the action of single electrical discharges
}

\author{
Mihaela Ețcu, Laurențiu Slătineanu* and Sebastian-Nicolae Croitoriu \\ Gheorghe Asachi Technical University of Iasi, Romania
}

\begin{abstract}
Currently, the electrical discharge machining is mainly applied to obtain surfaces difficult to be obtained using other machining processes in the workpieces made of electroconductive materials. The electroerosion process involves the initiation of electrical discharges in the zones where there is the lowest electrical resistance between the tool electrode and the workpiece. The study of the asperities peaks behavior under the action of the electric discharges would allow a better understanding of gradual developing of the surfaces generated by the electroerosive process. A way of addressing this problem could be based on the use of a device that would allow the study of the influence exerted by some process input factors on the results of a single electrical discharge developed between the test piece sharp peak and the tool electrode. The formulation of the functional requirements and the application of some methods of stimulating the technical creativity and of selecting solutions on the basis of predetermined criteria allowed the definition of a principle solution corresponding to an experimental device applicable for studying the behavior of sharp peaks under the action of single electrical discharges.
\end{abstract}

\section{Introduction}

A useful solution in the case of the necessity to obtain pieces of hard materials that are difficult to process by classical machining is to use one of the so-called unconventional machining. The difference between classical machining and unconventional machining, with the material removal from the workpiece, is based on the processes used to remove the machining allowance. In the classical machining methods, the basic principle used for the material removal from the workpiece is the principle of plastic deformation, while for the nonconventional machining methods, the working principle is based on the existence of developing thermal, chemical or micromechanical cutting processes $[1,2]$.

The electrical discharge machining (EDM) is the nonconventional machining process with material removal from the workpiece, based on the erosive effect of non-stationary (pulse) electric discharge, successively developed in the zones where there is the lowest electrical resistance between the tool electrode and the workpiece, when the size of the working gap has low enough values. As a result of an electrical discharge in a gas, microcavities are formed on the workpiece electrode (connected, for example, to the

${ }^{*}$ Corresponding author: slati@tcm.tuiasi.ro 
positive pole of a direct current electrical supply) and on the tool electrode (connected to the negative pole).

Analyzing the surface of a piece obtained by electroerosion, it can be seen that it is made up of a small microcavities chaining, each of the microcavities being the result of an electrical discharge occurred between the closest asperities existing on the surfaces of the two electrodes. It is possible that the action of electric discharge is influenced by the shape and dimensions of the surface asperities, which may

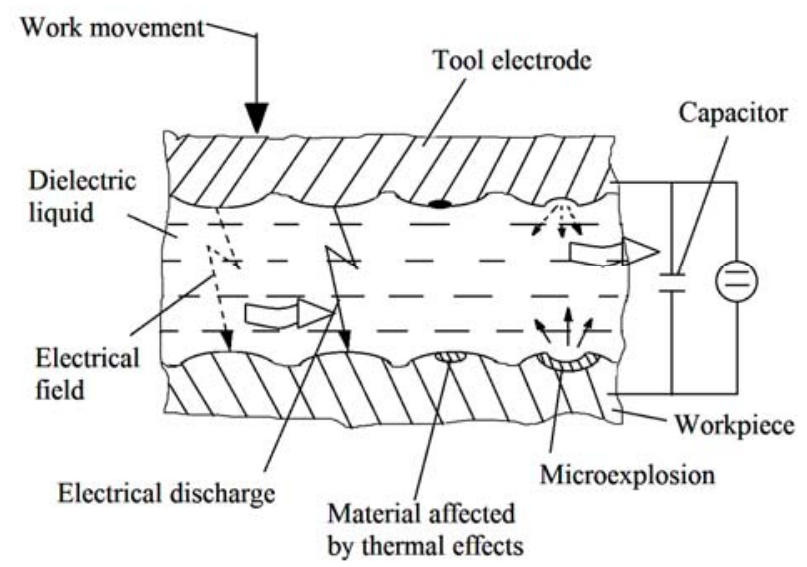

Fig. 1. Processes in the space between electrodes for electroerosion processing. lead to the hypothesis that the processes that develop at the level of the asperities peaks are influenced by their geometrical and dimensional characteristics. In the last decades, the researchers have shown interest in highlighting the extent to which different factors exert influence on the surface roughness obtained by electrical discharge machining.

Thus, Kiyak and Çakir carried out experimental research on the influence of pulse current, the pulse duration and the pause time between the pulses on the surface roughness parameter $R a$ that characterizes a surface obtained by an electroerosion process [3]. They appreciated that the negative influence of the tool electrode wear on the surface roughness cannot be avoided. Tang and Yang considered a simulation of the way in which the craters were formed in electroerosion process by using the finite element method [4]. They proposed a model based on the development of some thermo-hydraulic phenomena in the work gap. It has been appreciated that the efficiency of the material removal process in the workpiece is of about 0.04 . Shakeri et al. they used the neural network method to estimate the roughness of the surface that can be obtained by electroerosion with a wire electrode [5]. It was still possible to optimize processing conditions in terms of surface roughness and rate of material removal from the workpiece.

The objective pursued by the research presented in this paper was to highlight the extent to which the shape and dimensions of the surface asperities as well as other input factors in the electrical discharge machining can exert influence on the amount of material removed from the workpiece by a single electrical discharge.

\section{Considerations on the surface formation in electroerosion processing and simulation of this process}

It has previously been shown that the surface obtained by electroerosion can be considered as consisting of a microcavities chain, each of the microcavities being the result of an electrical discharge action. The electrical discharge machining process initially involves the nearing of the tool electrode to the workpiece, as a result of a specific work movement. For very small distances, an electric field appears between the very close asperities from the tool electrode and the workpiece (Figure 1). It is assumed that the electric discharge takes place for a distance $s$ between the electrodes which fulfills the condition: 


$$
s<\frac{U}{E}[\mathrm{~mm}],
$$

Where $U$ is the voltage applied to the electrodes, in $\mathrm{V}$, and $E$ is the dielectric rigidity of the medium in the gap, in $\mathrm{V} / \mathrm{mm}$ [6].

As the distance between the electrodes decreases, the electric field becomes very intense, and an electric discharge develops. In the contact areas of the electric discharge column with the electrode materials, a strong heating occurs, and the melting and evaporation of small quantities of the two electrode materials are possible. The rapid increase in material volume affected by the melting and vaporizing processes, as well as the emergence of the plasma column, lead to

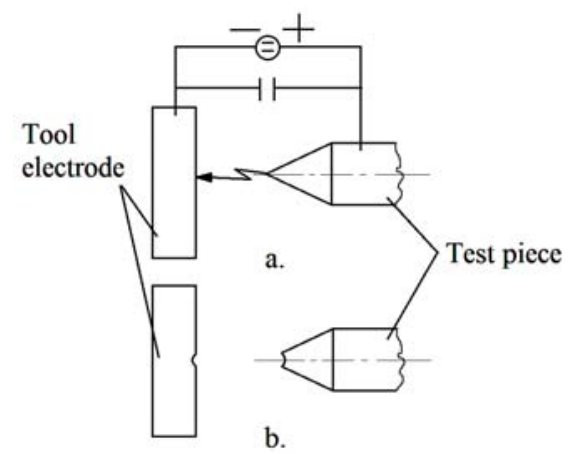

Fig. 2. Removal of material from electrodes when using a test sample with a conical tip: $a$ the situation prior to the electrical discharge; $b$ - after the electric discharge. the generation of microexplosions that contribute to the throwing of small amounts of the electrodes materials into the dielectric fluid. The circulation of the dielectric fluid into the space between the electrodes causes a removal of the detached particles from the two electrodes and the processes described above continue. The amount of the material removed from the workpiece electrode in a given unit of time gives an image of the productivity of the electrical discharge machining process.

It is expected that the shape and dimensions of an asperity exert influence on the amount of material removed from the workpiece by a single discharge. If, for example, one considers a conical asperity, it is possible that when the angle at the cone peak is higher, the heat generated by the discharge can quickly dissipate into a larger volume of material, which could result in a decrease of the amount of material removed from the asperity by a single electrical discharge. If the value of the cone angle is low, the electrical discharge should result in the removal of a larger amount of material from the asperity peak.

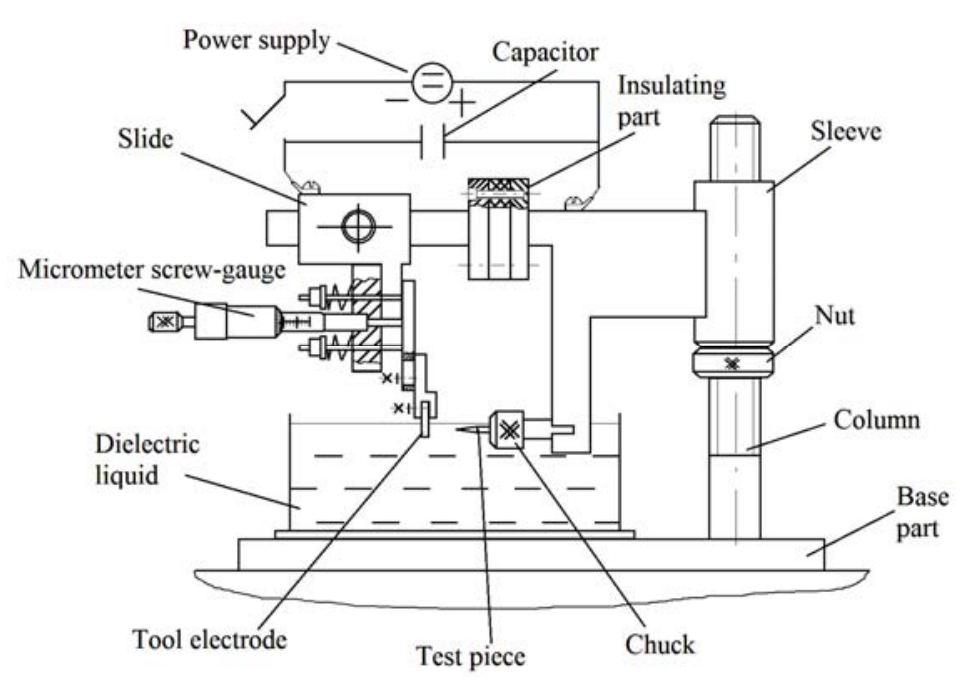

Fig. 3. Schematic representations of a device for the experimental investigation of the effects generated by a single electrical discharge on the test piece of the conical zone.
Based on the above mentioned considerations, a research could be developed to highlight the influence of distinct factors on a single asperity, simulated by the peak of a preset size cone (Figure 2). The generation of the electric discharge will result in the removal of a quantity of material from the test piece that has a conical peak, and by weighting the test piece before and after the electrical 
discharge, mass loss under the action of the single electrical discharge may be evaluated. Material removal rate will depend on the amount of energy consumed by electrical discharge:

$$
\mathrm{E}=\frac{C\left(U_{i}^{2}-U_{f}^{2}\right)}{2}[\mathrm{~J}],
$$

In this relation $C$ being the capacitance of the capacitors included in the discharge circuit, in $\mathrm{F}, U_{i}$ - the initial voltage applied to the capacitors and electrodes, in $\mathrm{V}, U_{f}$ - the value of the voltage remaining on the electrodes after the electrical discharge is finished, in $\mathrm{V}$.

Table 1. Functional requirements and design parameters in the case of the device for the study of sharp peaks behavior under the action of single electrical discharges.

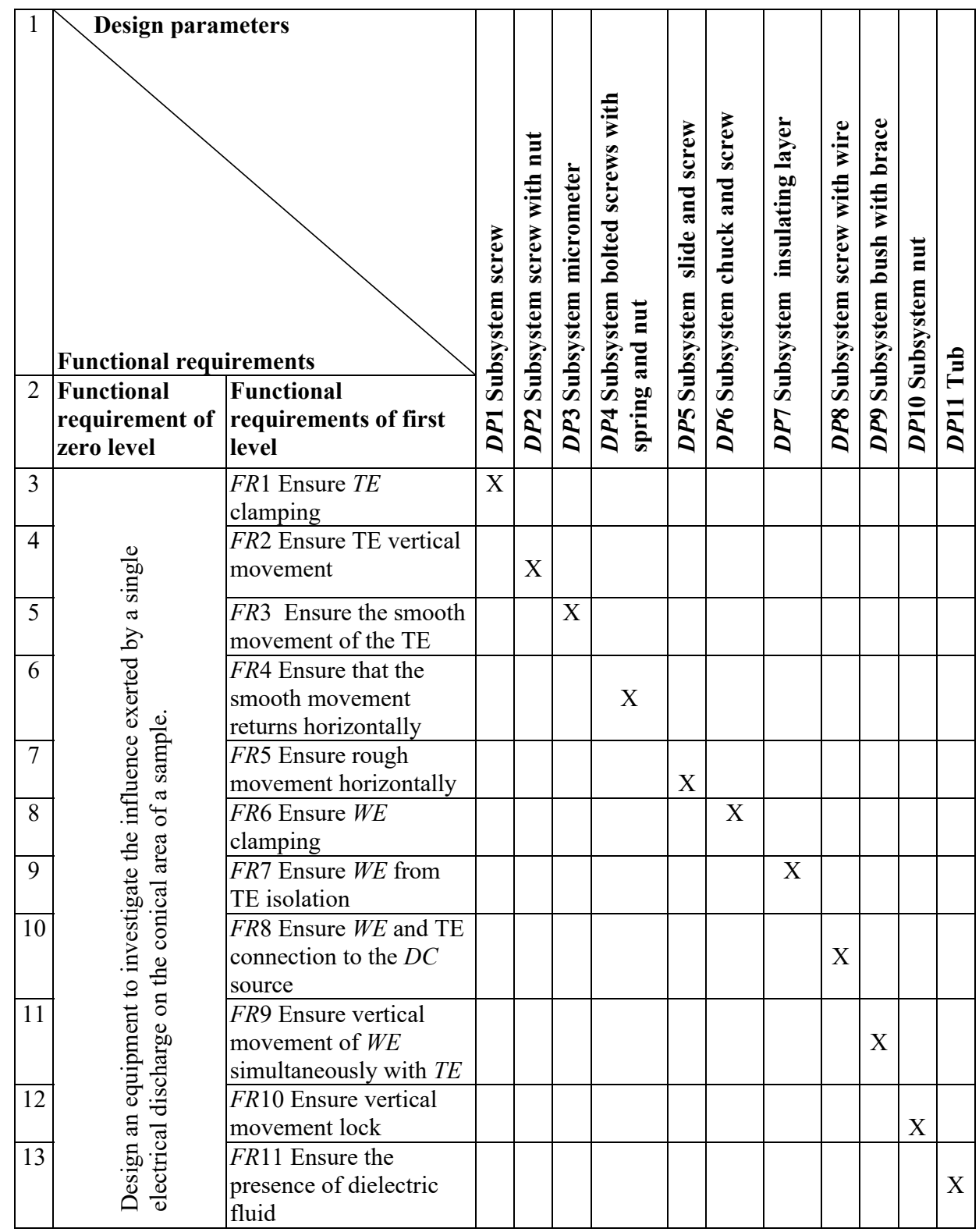


The materials used for tool electrodes must be characterized by a very good electrical and thermal conductivity, for which purpose the copper could be preferred. Other materials currently used to make tools electrodes are the brass, the aluminum, the graphite, the tungsten and some of its alloys, etc. The dielectric liquids could be based on the use of the mineral oil, petroleum, ethanol solutions, oil-in-water emulsions, distilled water, deionized water, etc. Some properties of the dielectric fluid (electrical resistance, viscosity, etc.) exert influence on the characteristics of the electric discharge, and also on the roughness of the surface obtained by electroerosion. The previous arguments have led to the conclusion of developing experimental attempts to verify some of the hypotheses previously formulated and to investigate some aspects of the effects of the single electric discharge on the asperities peaks that exist on the test piece surface.

\section{Possibilities for using the principles of axiomatic design in establishing a device}

The identification of a solution for the device whose use could highlight the effects of a single electric discharge on the test pieces that have conical peaks could be possible by applying some methods of stimulating creativity. Methods such as the ideas diagram, the morphological matrix method, the axiomatic design method can be used for this purpose [710]. Subsequently it has been preferred to apply some principles of the axiomatic design for operative contouring of a solution for the proposed device.

The axiomatic design was proposed in the 1970s by the Professor Nam Pyo Suh, while he was working at the Massachusetts Institute of Technology [9-10]. In principle, the axiomatic design accepts the validity of two axioms, namely the axiom of the independence of functional requirements and the axiom of using a minimal amount of information. In order to apply the axiomatic design, firstly the so-called functional requirements $(F R)$ were defined, and at a later stage, the identification of those subassemblies or components of the proposed device that can materialize each of the functional requirements were considered. In this second stage, in fact, the so-called design parameters $(D P)$ are established.

A more operative application and also a suggestive illustration of the correspondence between $F R$ functional requirements and $D P$ design parameters can be achieved by using a design matrix. An example of a mathematical design matrix valid for the proposed device can be seen in Table 1. In this table, along the lines, the main $F R$ functional requirements, along the columns, have the $D P$ design parameters.

By taking into account a larger number of functional requirements, this array becomes more complex and it can better highlight those components and subassemblies necessary to ensure the use of the device under the conditions specified above.

\section{Proposed principle solution}

The version of the device identified by taking into account the functional requirements and the design parameters according to the succinct information presented in Table 1 can be seen in figure 3 . One can notice the use of a moveable sleeve and a nut to immobilize the sleeve on a threaded vertical column. The sleeve is solidarized with a main plate on which one can move and clamp a horizontal support rod for placing the tool electrode. The reduction of the distance between the tool electrode and the test piece that have a conical zone until the initiation of the electrical discharge is done by means of a micrometer. The test sample is mounted in a chuck assembled to the main plate. The tool electrode and the test piece are connected to a discharge circuit powered by a direct current supply. The 
change of the single electrical discharge energy can be achieved by modifying the capacity of some capacitors included in the discharge circuit.

The possible stages of the generating the single discharge could be: 1 . Charging the capacitors, keeping the distance between the tool electrode and test piece so that a discharge could not be initiated; 2. Interruption of the power supply; 3 . Decrease o the distance between electrodes to initiate the electrical discharge. One could take into consideration the delay time, which depends on the gap size, the dielectric conductivity, and the ignition voltage.

To highlight the influence exerted by the dielectric on the effect of the single electrical discharge, a tank was provided. In this tank, distinct dielectric fluids could be introduced. By moving the sleeve along the vertical column, it is possible to insert the tool electrode and the test sample into the dielectric fluid in which a single electrical discharge is to be initiated.

\section{Conclusions}

A surface obtained by electroerosion can be considered to be composed of a microcavities chain generated by the electrical discharges generated between the closest asperities on the surfaces of the tool electrode and the workpiece. A simulation of the way in which an asperity from the workpiece surface of the workpiece could be affected is possible by generating a single electrical discharge between the surface of the tool electrode and the conical area of a sample. Studying the effects of electrical discharge on the conical area of the sample can provide additional information on how to generate a surface by electroerosion. To materialize such a scientific investigation, principles of axiomatic design were used to develop a principal device solution that would provide conditions for producing electrical discharge between a tool electrode and a sample having a conical area. In the future, it is envisaged to realize this device and conduct an experimental investigation on the influence of different factors on the sampling of material from the conical sample, as a result of a single electrical discharge.

\section{References}

1. O. Dodun, Nonconventional technologies. Machining with materialized tools (in Romanian) (Tehnica-Info Publishing House, 2001)

2. L. Slătineanu, G. Nagît, O. Dodun, M. Coteaţă, F. Chinesta, A. Gonçalves-Coelho, J. Pamies Teixeira, M. San Juan, L. Santo, F. Santos, Non-traditional manufacturing processes (Tehnica Info Publishing House, Chişinău, 2004)

3. M. Kiyak, O. Çakir, J. Mater. Process. Tech. 191, 1-3, 141-144 (2007)

4. J. Tang, X. Yang, Procedia CIRP, 42, 685-690 (2016)

5. S. Shakeri, A. Ghassemi, M. Hassani, A. Hajian, Int. J. Adv. Manuf. Technol. 82, 549557 (2016)

6. B.A. Artamonov et al. Dimensional electrical machining of the metallic materials. Handbook (in Russian) (Vysshaia shkola, Moskow, 1978)

7. V. Belous, Inventics (in Romanian) (Editura Asachi, Iaşi, 1992)

8. L. Slătineanu, Industrial property (Performantica Publishing House, 2013)

9. N.P. Suh, The principles of design (Oxford University Press, New York, 1990)

10. N.P. Suh, Axiomatic Design: Advances and Applications (Oxford University Press, New York, 2001) 